\title{
Laminar Organization and Contrast Sensitivity of Direction-Selective Cells in the Striate Cortex of the Old World Monkey
}

\author{
M. J. Hawken,' A. J. Parker,' and J. S. Lund ${ }^{2}$ \\ University Laboratory of Physiology, Oxford OX13PT, United Kingdom, and 'Department of Psychiatry, University of \\ Pittsburgh, Pittsburgh, Pennsylvania 15261
}

\begin{abstract}
The directional preference of neurons sampled from all layers of the striate cortex was determined using the responses to drifting grating stimuli of optimal spatial and temporal frequency. In addition, contrast sensitivity as a function of spatial frequency was measured and from the resulting spatial contrast sensitivity function the peak contrast sensitivity and optimal spatial frequency were obtained. The distribution of directionally selective cells showed a distinct laminar pattern. Upper layer 4 (4a, 4b, and $4 c \alpha$ ) and layer 6 were the only cortical layers with neurons that showed a pronounced preference for the direction of stimulus motion. The directionally selective cells in these layers are among those with the highest contrast sensitivities but had optimal spatial frequencies that were confined to the low and middle range of the optimal spatial frequency distribution. These findings suggest that the directionally selective cells may fall into at least 2 distinct populations, which may be the first stages in the visual pathway that correspond to those channels, inferred from psychophysical experiments, that underlie the detection of movement.
\end{abstract}

The striate cortex (V1) is the first location in the visual pathway of primates where neurons show directional selectivity (Hubel and Wiesel, 1968). It is these directionally selective cells that are most likely to provide the initial properties for the "channels" or "mechanisms" that are responsible for the analysis of the direction of moving objects in perception. The interrelationship between $\mathrm{V} 1$ and specialized extrastriate visual areas has become of considerable importance in understanding the functional divisions underlying visual processing. It is now well established that MT (or V5), one of the extrastriate visual areas located on the posterior bank of the superior temporal sulcus, has a large number of direction-selective neurons (Dubner and Zeki, 1971; Zeki, 1974; Van Essen et al., 1981; Albright, 1984), and it is thought that MT is primarily involved in processing information concerned with a number of aspects of visual motion (Movshon et al., 1985; Newsome et al., 1985; Mikami et al., 1986a, b). Since much of the input to MT is obtained via inputs from the geniculostriate pathway, then it is likely that the functional properties of cells in MT are determined, to some

Received Aug. 10, 1987; revised Feb. 1, 1988; accepted Feb. 2, 1988.

This research was supported by MRC Grant 7900491 to C. Blakemore, NATO Grant 85/0167, Wellcome Trust major equipment grant, and USAF Grant AFOSR85-0296.

Correspondence should be addressed to M. J. Hawken, University Laboratory of Physiology, Parks Road, Oxford OX1 3PT, UK.

Copyright (C) 1988 Society for Neuroscience $0270-6474 / 88 / 103541-08 \$ 02.00 / 0$ extent, by the properties of cells in V1 which project to MT. The major direct projections from Vl to area MT (Zeki, 1969) arise from 2 distinct cortical layers (Lund et al., 1976; Maunsell and Van Essen, 1983): one of these is layer 4b, where the projection is derived from patches or groups of cells separated by patches of nonprojecting cells (Shipp and Zeki, 1985), while the other is layer 6 (Fries et al., 1985).

Most studies of the functional organization of V1 in the primate report that cells with directionally selective receptive fields are found in all cortical layers, except maybe layer $4 \mathrm{c}$. However, unlike the cat's striate cortex, where there is a reasonably uniform distribution of directionally selective cells throughout all cortical layers (Gilbert, 1977), in monkey V1 there is an uneven laminar distribution. Although there is agreement in the literature of a relatively high concentration of directionally selective cells in layers $4 \mathrm{~b}$ and 6 (Hubel and Wiesel, 1968; Dow, 1974; Livingstone and Hubel, 1984; Orban et al., 1986), as yet there has not been a quantitative assessment of the laminar distribution throughout all the cortical layers to compare with Gilbert's (1977) analysis in the cat.

In the analysis of stimulus motion, psychophysical experiments indicate that the threshold detection of moving patterns is performed by oriented, directionally tuned mechanisms (Sekuler and Ganz, 1963; Pantle and Sekuler, 1968; Sharpe and Tolhurst, 1973). Of particular interest is the observation that contrast thresholds for stimulus detection alone and for the identification of the direction of motion are similar (Levinson and Sekuler, 1975; Watson et al., 1980). Furthermore, the channels from which information is combined to signal the perceived direction of complex patterns behave like the oriented, direction-selective channels described above (Adelson and Movshon, 1982). It might be expected then that some of the directionally tuned neurons found in V1 would also have low contrast thresholds, if they are to be considered as the initial substrate for the psychophysically identified motion channels. Consequently, we have also compared the contrast sensitivities of directional and nondirectional V1 neurons.

\section{Materials and Methods}

Experiments were carried out on 7 adult Old World monkeys, 6 Macaca fascicularis, and 1 Cercopithicus aethiops. Animals were initially anesthetized with ketamine hydrochloride before insertion of a venous cannula; then, for the ensuing surgery, steroid anesthetic (Saffan: alphaloxone and alphadolone acetate) was used. All incisions were infiltrated with long-lasting local anesthetic (Marcain). For recording, animals were given an initial loading dose of barbiturate anesthetic (Sagatal: $6 \mathrm{mg}$ / $\mathrm{kg}$ ) and muscle relaxant (pancuronium bromide: $0.2 \mathrm{mg} / \mathrm{kg}$ ). To maintain anesthesia and paralysis, a continuous infusion of barbiturate (1.5$3.0 \mathrm{mg} / \mathrm{kg} / \mathrm{hr}$ ) and muscle relaxant was given in a mixture of glucose 
NON-DIRECTIONAL

A
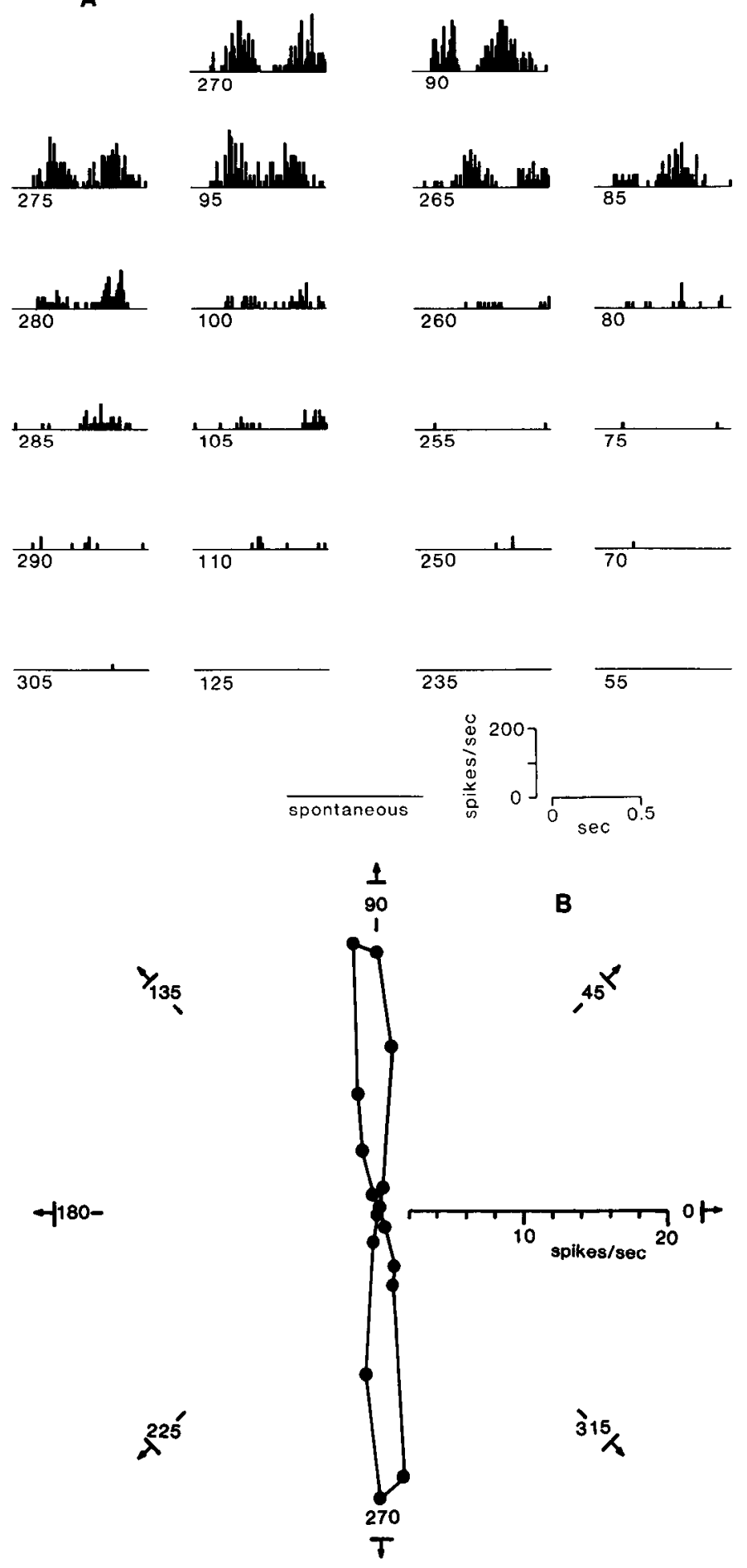

Figure 1. Orientation tuning $(A, B)$ of a simple cell without direction preference recorded in layer 2 . $A$, Peristimulus time histograms for a range of orientations and for both directions of drift at each orientation. The top pair of histograms show the response elicited by 2 temporal cycles of a horizontally oriented grating moving either downward (left histogram, marked $270^{\circ}$ ) or upward (right histogram, marked $90^{\circ}$ ). In subsequent rows showing 2 pairs of histograms, the stimulus orientation is the same for each of the histograms in a pair, but movement is in opposite directions. This is denoted by the values showing the orientation and dircction bclow cach histogram, which are $180^{\circ}$ apart for each pair. Averaged responses derived from $A$ (see text) are plotted on polar coordinates to give both the orientation and the direction tuning and saline. Electrocardiogram (ECG) and electroencephalogram (EEG) were monitored continuously, and anesthetic state was judged to be satisfactory if there was almost complete slow-wave EEG activity and if potentially mildly noxious stimuli produced no change in the EEG or heart rate. Supplementary anesthetic doses were given, if required, to maintain anesthetic state. Temperature was maintained at 37.5$38.0^{\circ} \mathrm{C}$ and expired $\mathrm{CO}_{2}$ between $4.5-5.5 \%$. The detailed methods of preparation, recording, data collection, and analysis have been recently described elsewhere (Hawken and Parker, 1984, 1987).

Extracellular action potentials were recorded with glass-coated tungsten microelectrodes (Merrill and Ainsworth, 1972) with 4-8 $\mu \mathrm{m}$ exposed tips lowered into the cortex through a small craniotomy and durotomy with a stepping-motor microdrive. For initial classification, receptive field properties were investigated with hand-held stimuli backprojected onto a screen $171 \mathrm{~cm}$ in front of the animal. Light or dark bars whose length and width could be varied and that could be projected with white or colored (broad-band Wratten filters) light were used as stimuli. Following this initial classification for cell type, ocular dominance, and orientation preference, the receptive field was centered on a CRT screen (Joyce Electronics) with a P4 phosphor and a mean luminance of $280 \mathrm{~cd} / \mathrm{m}^{2}$ upon which grating stimuli generated under computer control were displayed for quantitative determination of responses and sensitivity. The procedures for the measurement of spatial frequency tuning, linearity of spatial summation, orientation and direction tuning, and contrast sensitivity have all been described elsewhere (Hawken and Parker, 1987; Parker and Hawken, 1988).

During each oblique electrode penetration we made between 3 and 5 small electrolytic lesions (2-3 $\mu \mathrm{A}$ for $3-5 \mathrm{sec}$ ) to mark the tip of the recording electrode; these lesions were used in subsequent histological reconstruction of the electrode penetration and to assign each recorded cell to a cortical layer. At the end of each experiment, animals were killed with an overdose of anesthetic and then perfused through the heart with a mixture of heparinized saline, followed by 2 liters of fixative (1.25\% paraformaldehyde $+2.5 \%$ glutaraldehyde in phosphate buffer, $\mathrm{pH}$ 7.4). Brains were blocked with the skull reinserted in the stereotaxic frame, so that cuts could be made parallel to the electrode penetrations; then the blocks were removed and immersed in 30\% sucrose-saline until they sank. Frozen sections were cut $(50 \mu \mathrm{m})$, washed, and stained for the mitochondrial enzyme cytochrome oxidase (Wong-Riley, 1979), then counterstained for Nissl with cresyl violet. From these scetions wc located the lesions and reconstructed the penetrations.

\section{Results}

We were able to ascertain the receptive field properties with hand-held stimuli for a total of 285 cells, ranging between 31 and 52 neurons per animal, and obtained quantitative orientation tuning curves on just over half the cells from this sample. Apart from those cells lost during quantitative recording, the majority of the remaining cells classified with hand-held stimuli but not quantitatively analyzed either could not be adequately driven (because of pronounced color preferences or they required contrasts $>0.7$ to generate modest responses) or they showed very strong end-stopping (and our equipment did not allow easy measurement of the orientation tuning of strongly end-stopped cells). Here, then, we report on the responses of 147 neurons.

For each neuron, directional preference was determined from the responses to drifting sinusoidal grating stimuli, whose spatial frequency was nearly optimal. The response was determined for a range of orientations around the preferred orientation and for both directions of drift. For nonoriented cells (total of 12 out of 147) and cells broadly tuned for orientation, the response was measured at $20^{\circ}$ intervals throughout the full $360^{\circ}$. The temporal frequency was adjusted to be nearly optimal, once the

of the cell's receptive field. For this cell, at all orientations tested, the response to opposite directions of motion are almost equal in magnitude; at the preferred orientation, the directional index is 1.10 . 
preferred spatial frequency and orientation had been determined.

Figure $1 A$ shows the orientation tuning histograms from a simple cell in layer 2 of V1. Each histogram shows the response to 2 complete temporal cycles of the grating drifting over the cell's receptive field, and, as would be expected for a simple cell, there is a dominant response modulation at the fundamental frequency. The cell shows a clear preference for a horizontally oriented grating but is equally responsive to either upward $\left(90^{\circ}\right)$ or downward $\left(270^{\circ}\right)$ movement. Although the response of the cell declines either side of the preferred orientation, there is little or no change in the direction preference at nonoptimal oricntations. This can be seen most clearly from the histograms sharing the same nonoptimal orientation and differing only in the direction of drift, for example, those marked $280^{\circ}$ and $100^{\circ}$ or those marked $260^{\circ}$ and $80^{\circ}$ in Figure $1 \mathrm{~A}$.

Figure $2 A$ shows the histograms obtained from a simple cell recorded in layer 6 . This neuron also shows a clear orientation preference, but unlike the cell whose responses are illustrated in Figure $1 A$, it has a pronounced directional preference as well. For a number of the directionally selective cells, there was an initial transient at the onset of the moving grating, which is shown quite clearly at some orientations in Figure $2 A$, those between $50^{\circ}$ and $65^{\circ}$ in the nonpreferred direction and $230^{\circ}-$ $240^{\circ}$ in the preferred. The onset transient is not a strongly direction-selective component of the response, but occurs only at some orientations. At the orientations where the onset transient is evident there is a much reduccd rcsponsc to the sccond cycle of a grating moving in the null direction. Since the onset transient is orientation dependent, it is most likely to be analogous to the response to flashed or flickered gratings seen in the "null" phase test. We presume that the spatial position of the grating at those orientations where the onset transient is produced is equivalent to finding the optimal spatial phase in the "null" phase test.

Figures $1 B$ and $2 B$ show the orientation tuning functions constructed from the responses of these 2 cells. We have used the total elevation in discharge, minus the background, over the temporal modulation of the discharge ( $f 0$ component) as the measure of response for all complex cells and for simple cells where there is little or no spontaneous activity. For simple cells with an appreciable spontaneous discharge rate, we used the temporal modulation of the discharge ( $f 1$ component) as the response measure. The preference for direction was calculated as the ratio of the response in the optimal direction to the response in the opposite direction, at the orientation that gave the maximal response in the preferred direction. On this scale, a value of 1.0 indicates no preference for direction, while a high value of the ratio indicates a pronounced directional preference. This scale was adopted by Gilbert (1977) in his study of the directional preference in different layers of the cat's striate cortex. A response ratio of 2:1 seems to have been agreed upon as a reasonable value to use in dividing directionally selective from nonselective cells (Gilbert, 1977; DeValois et al., 1982a). The directional index for the cell whose responses are illustrated in Figure $1, A, B$, is 1.10 , a value close to one, which means the response is essentially nondirectional. The directional index for the neuron whose responses are shown in Figure 2, $A, B$, is 11.8, an example of a cell with a strong directional preference. By adopting this method of assigning a single quantitative value to the directional preference, the whole sample could be examined with respect to the relative position within each cortical layer.

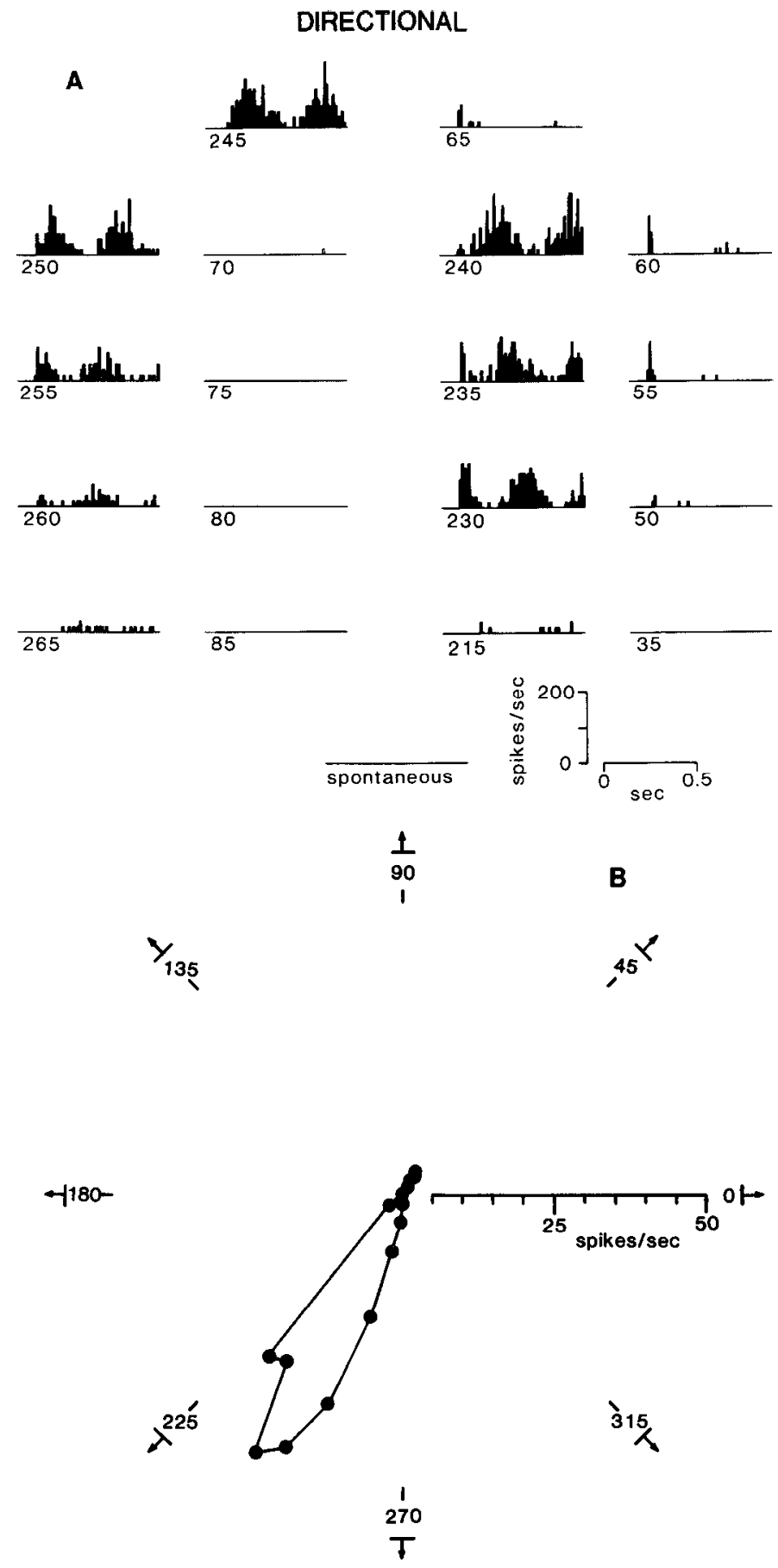

Figure 2. Orientation tuning $(A, B)$ of a simple cell with strong direction selectivity recorded in layer 6 . The histograms in $A$ show the response to 2 cycles of the grating passing the cell's receptive field. Each pair of histograms is for the same stimulus orientation and for opposite directions of drift. For this cell the left-hand histograms of each pair are for downward and leftward motion, while the right-hand histograms are for the opposite direction-upward and to the right. $B$, Responses plotted in polar coordinates, as described in the legend to Figure 1. Direction index, 11.8 (indicating a strong preference for the grating direction).

\section{Laminar position}

Oblique electrode penetrations, marked by $3-5$ lesions, were reconstructed from sections that were stained for cytochrome oxidase and counterstained for Nissl substance. The boundaries of the cortical layers along with the reconstructions of the elec- 


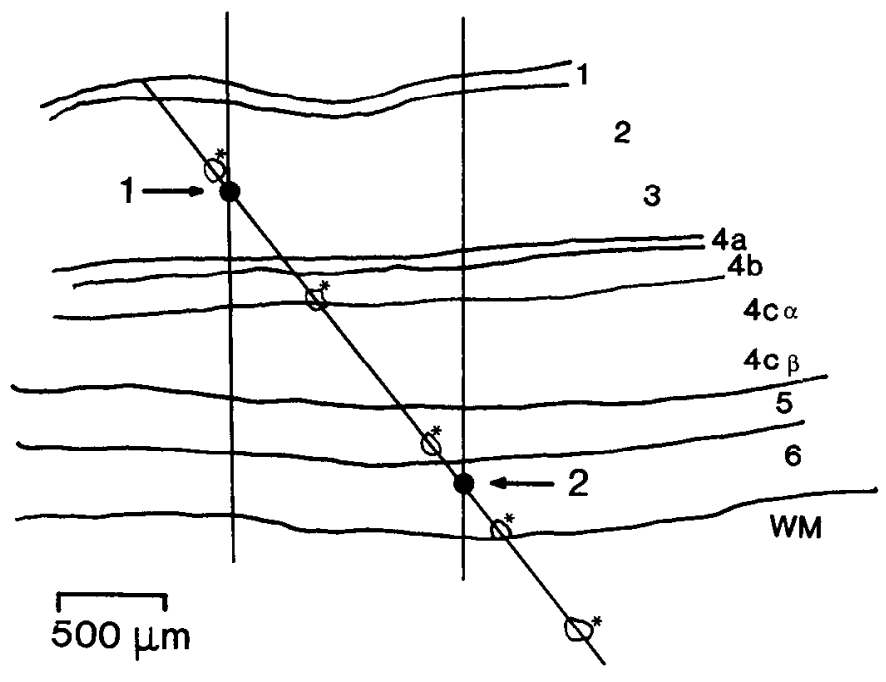

Figure 3. Schematic reconstruction of an oblique penetration through $\mathrm{V} 1$, showing the positions of the 5 electrolytic lesions (asterisks) made during the penetration. The filled circles, marked $I$ and 2 , are the positions of 2 cells, and the surface normal lines through them are used to obtain a relative depth for each recorded cell within its layer as described in the text.

trode penetrations were drawn, using a camera lucida, from the stained sections. The schematic drawing of one such reconstructed penetration is shown in Figure 3. Because penetrations were made obliquely and the thickness of the cortex, and indeed of individual layers, varies in different sections of the striate cortex, the following procedure was adopted to assign the recording site of each neuron to a "relative depth within a lamina." First, the position of each neuron's recording site, using the nearest lesion as a reference, was marked on the reconstruction. Following this, a surface normal line was projected through the estimated recording site of each cell. The total thickness of the layer (or sublayer), in which the recording site was positioned, was measured along the projected surface normal line, as well as the position of the cell along the same line. From these measurements a relative depth within the layer was determined for each cell's recording site.

Some of the salient features of this procedure arc shown for 2 cells recorded in the same oblique electrode penetration illustrated in Figure 3. A nondirectionally selective cell (direction index 1.53) was recorded at the position marked 1 and a directionally selective cell (direction index 6.55) at the position marked 2 . The recording site marked 1 in Figure 3 is $392 \mu \mathrm{m}$ from the border of layers 1 and 2, while the total thickness of layers 2 and 3 is $703 \mu \mathrm{m}$ along the surface normal line. Therefore, the relative depth is 0.56 within the combined thickness of layers 2 and 3 . The site marked 2 in layer 6 is $95 \mu \mathrm{m}$ from the border of layers 5 and 6 ; layer 6 has a depth of $351 \mu \mathrm{m}$ on the surface normal, so this cell's relative depth in layer 6 is 0.27 . This procedure was followed to determine the position of each of the cells shown in Figure 4.

There is some variation in the thickness of each cortical layer or sublayer both within a single parasagittal section and in different sections, probably reflecting real differences in thickness across the cortex. To estimate the mean thickness of each layer as a proportion of the total vertical distance from cortical surface to white matter, the vertical extent of each layer was measured in 2 surface normal projections from each of the sections con-

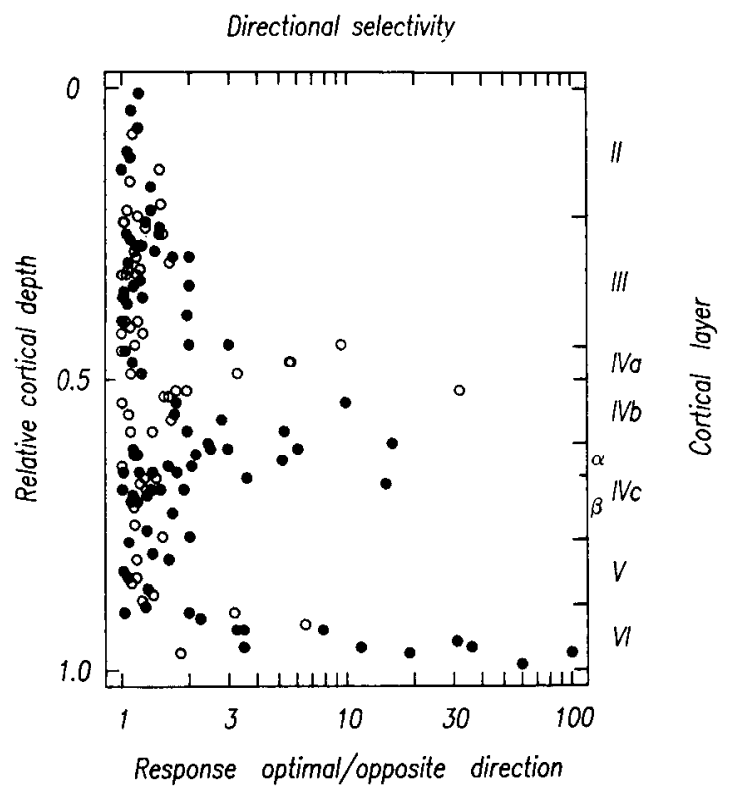

Figure 4. Directional preference of 147 cells is plotted as a function of laminar position. Directional preference calculated as ratio of peak response in the preferred direction to the response in the nonpreferred direction. Only cells in layer 6 and middle layer 4 show pronounced directional tuning. Twelve of the cells included in this distribution were nonoriented. Open symbols, 54 complex cells plus 3 nonlinear, nonoriented cells; closed symbols, 81 simple cells plus 9 linear nonoriented cells.

taining the electrode penetrations. This gives an average thickness for each layer, which is the value used in Figure 4 along the vertical axis. This estimate was made so that a realistic representation of the proportion of total cortical depth assigned to each cortical layer could be shown in Figure 4.

From the combined cytochrome oxidase and Nissl-stained sections it is moderately straightforward to distinguish the borders between layers $1 / 2,3 / 4 a, 4 a / 4 b, 4 b / 4 c \alpha, 4 c \beta / 5,5 / 6$, $6 /$ white matter. On the other hand, we had no anatomical criteria, from our material, to distinguish the borders between layers 2 and 3 or between $4 \mathrm{c} \alpha$ and $4 \mathrm{c} \beta$. As will be seen in the next section, there does not appear to be any sharp division of the properties of cells in layers 2 and 3 in terms of their directional preferences. The division between $4 c \alpha$ and $4 c \beta$ is, however, important both anatomically and functionally. It is quite clear that neurons from the magnocellular layers of the dorsal lateral geniculate nucleus (LGN) terminate in the upper portion of $4 c, \alpha$ subdivision, while those from the parvocellular layers terminate in the lower region, $\beta$ subdivision (Hubel and Wiesel, 1972; Hendrickson et al., 1978; Blasdel and Lund, 1983). We have used the autoradiographic distribution of labeled terminals from the study of Hendrickson et al. (1978) to estimate the relative proportion of layer $4 \mathrm{c}$ to assign to the $\alpha$ and $\beta$ subdivisions. From their results, the upper third of layer $4 \mathrm{c}$ is occupied by terminals from the magnocellular layers of the LGN, while the lower two-thirds is occupied by parvocellular terminals; we have therefore chosen this division as the boundary in this study. Nonetheless, there is most likely an overlap of terminal branches between magnoccllular and parvocellular projections to layer $4 c$; furthermore, the division of layer $4 c$ in the ratio of $2: 1$ (parvo: magno) is unlikely to be exact. So there are 2 likely sources of positional inaccuracy: one in the definition of the laminar boundaries and a second resulting from interpolation 

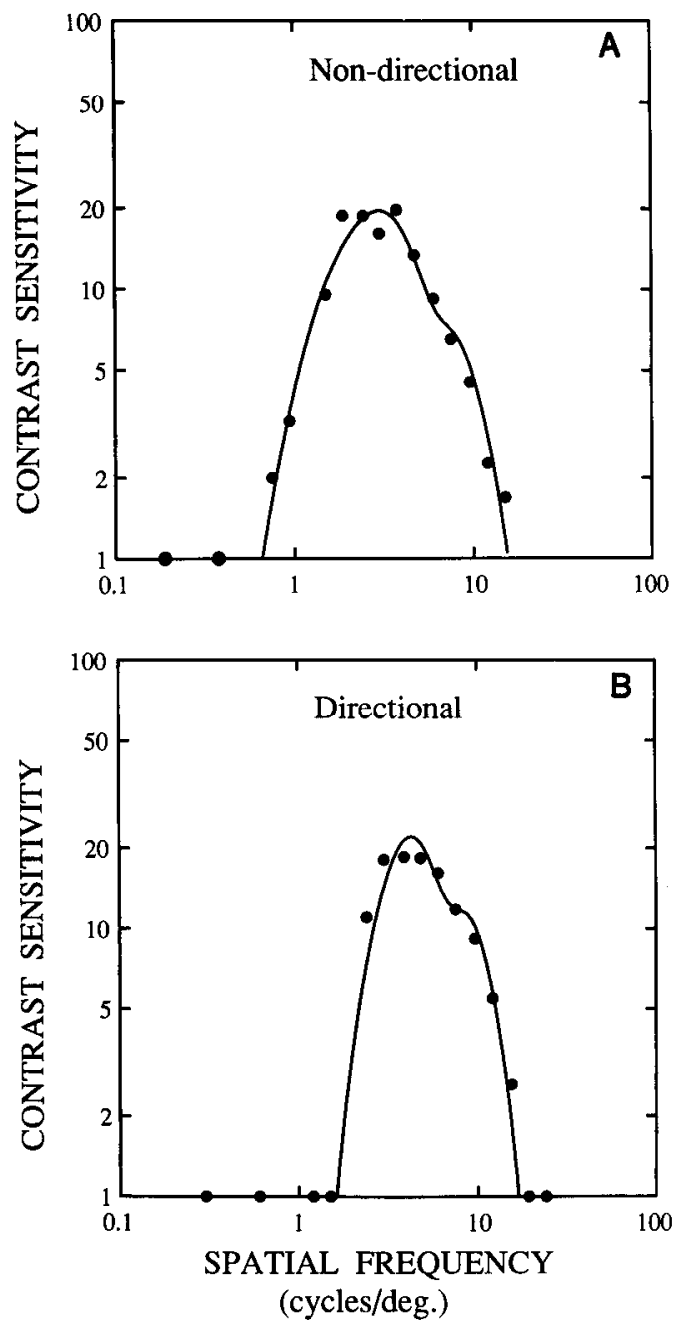

Figure 5. Contrast threshold was determined for a range of spatial frequencies for the cells shown in Figures 1 and 2. Each closed symbol shows the mean value of contrast sensitivity obtained from 12 independent determinations of the threshold via a staircase procedure. The peak contrast sensitivity was taken as the peak of the fitted d-DOG-s function, shown by the smooth curve through the sensitivity measurements. The function is specified by the equation

$$
\begin{aligned}
k_{c_{1}} \exp [ & \left.\left(x / x_{c_{1}}\right)^{2}\right]-k_{s_{1}} \exp \left[-\left(x / x_{s_{1}}\right)^{2}\right] \\
- & (1-g)\left[k_{c_{2}} \exp \left[-\left((x+S) / x_{c_{2}}\right)^{2}\right]-k_{s_{2}} \exp \left[-\left((x+S) / x_{s_{2}}\right)^{2}\right]\right] \\
- & g\left[k_{c_{2}} \exp \left[-\left((x-S) / x_{c_{2}}\right)^{2}\right]-k_{s_{2}} \exp \left[-\left((x-S) / x_{s_{2}}\right)^{2}\right]\right]
\end{aligned}
$$

In this equation, $x$ is position in Cartesian coordinates; $x_{r_{1}}, x_{t_{2}}, x_{r_{1}}$, and $x_{s_{2}}$ are the space constants of the Gaussians; and $k_{c_{1}}, k_{c_{2}}, k_{s_{1}}$, and $k_{s_{2}}$ control the sensitivity of the Gaussians; $g$ determines the surround symmetry; and $S$, the separation of the DOG mechanisms (Hawken and Parker, 1987). $A$, The cell whose orientation and directional tuning are shown in Figure 1. The orientation of the grating used to make the contrast sensitivity mcasurements was horizontal, and it moved downward at $2.6 \mathrm{~Hz}$. The smooth curve is the best-fitting d-DOG-s function, whose parameters are

$$
\begin{aligned}
& x_{c_{1}}=2.02, x_{s_{1}}=19.6, x_{c_{2}}=4.48, x_{s_{2}}=25.1, S=8.49, \\
& k_{c_{1}}=240, k_{s_{1}}=17.4, k_{c_{2}}=92.3, k_{s_{2}}=10.6, g=0.5 .
\end{aligned}
$$

$B$, The spatial contrast sensitivity function for the directionally selective simple cell whose details are given in the legend to Figure 2. For the sensitivity measurements the gratings moved down and to the left. The smooth curve is the best-fitting d-DOG-s function whose parameters are

$$
\begin{aligned}
& x_{c_{1}}=2.14, x_{s_{1}}=3.85, x_{c_{2}}=4.12, x_{s_{2}}=6.32, S=7.38 \\
& k_{c_{1}}=573, k_{s_{1}}=318, k_{c_{2}}=297, k_{s_{2}}=194, g=0.5 .
\end{aligned}
$$

between electrolytic lesions within a penetration. Thus, for a thin layer such as $4 \mathrm{a}$, the error in positional assignment will be large compared with other layers.

\section{Directional preference in different layers}

Figure 4 shows the directional index of each cell plotted against its relative depth within its layer. The majority of cells with a directional index $>2$ are confined to the upper sublayers of 4 and layer 6 . There are no cells that show a pronounced directional preference in the upper three-quarters of layers $2+3$, layer $4 c \beta$, or layer 5 . Comparing the layers just on the basis of the proportion of cells with directional indices of 2.0 or greater, layer 6 has the highest proportion of neurons with a directional bias $(72 \%)$, followed by layers $4 \mathrm{c} \alpha(47 \%), 4 \mathrm{a}(42 \%)$, and $4 \mathrm{~b}$ $(25 \%)$, but there is less certainty in locating cells in layer $4 \mathrm{a}$ than in other layers. Therefore, some of the directional cells assigned to reside in $4 \mathrm{a}$ might be within $4 \mathrm{~b}$. Without a much larger sample we can only conclude that the directional cells in upper layer 4 are definitely found within $4 \mathrm{~b}$ and $4 \mathrm{c} \alpha$ and most probably, but not certainly, within $4 a$. Furthermore, the exact frequency of directional cells must be interpreted with caution because there is no information available to estimate the possible electrode biases with respect to selecting direction-selective cells within V1.

The preponderance of cells with a directional preference in layer 6 is in accord with previous findings (Schiller et al., 1976; Livingstone and Hubel, 1984; Orban et al., 1986). Whereas Schiller et al. (1976) found both simple and complex types among the layer 6 cells, Livingstone and Hubel (1984) found only complex cells in layer 6 and simple cells in $4 \mathrm{~b}$. Using a criterion based on linearity of spatial summation to distinguish simple and complex cells (Movshon et al., 1978; Hawken and Parker, 1987), in this study both cell types were found in layers $4 \mathrm{~b}$ and 6 . In fact, in layer 6 there are more simple cells than complex ones. Furthermore, among the directional cells, again seen most strongly in layer 6 , there is a tendency for simple rather than complex cell type. Of the nonoriented cells, 9 showed linear spatial summation and 3 nonlinear spatial summation, and obviously, by definition, they are not directionally selective. Therefore, we have included them in the simple/complex categories on the basis of their spatial summation properties, but they would, of course, be a separate group with respect to other characteristics such as orientation.

DeValois et al. (1982a) studied the distribution of directionally selective cells in V1 with respect to a number of factors other than cortical layer. In their study there were no significant differences between the number of cells in the foveal and parafoveal regions with respect to direction selectivity. From a total sample of 222 cells, they found $27 \%$ showed a directional preference ( $>2.0$ on the scale used in this paper), and, albeit with a smaller sample, we find $28 \%$ of cells have a directional index of 2 or greater, foveal and perifoveal groups being similar at $29 \%$ and $26 \%$, respectively. Using bar stimuli, Schiller et al. (1976) found $35 \%$ of cells in V1 showed a directional preference, while in Orban et al.'s (1986) "central" sample, $27 \%$ of neurons were directional. Therefore, no matter what method is used to assess directionality it seems that between a quarter and a third

The units for $x_{c_{1}}, x_{c_{2}}, x_{s_{1}}$, and $x_{s_{2}}$ and $S$ are minutes arc and for $k_{c_{1}}, k_{c_{2}}$, $k_{s_{1}}$, and $k_{s_{2}}$ are contrast ${ }^{-1}$ (minutes arc) $)^{-1}$ 
Figure 6. Distribution of peak contrast sensitivity for directional and nondirectional neurons. $A$, Neurons in the foveal group, receptive field centers less than $1.5^{\circ}$ eccentric. $B$, Perifoveal group, with fields $1.5^{\circ}-3.5^{\circ}$ eccentric.

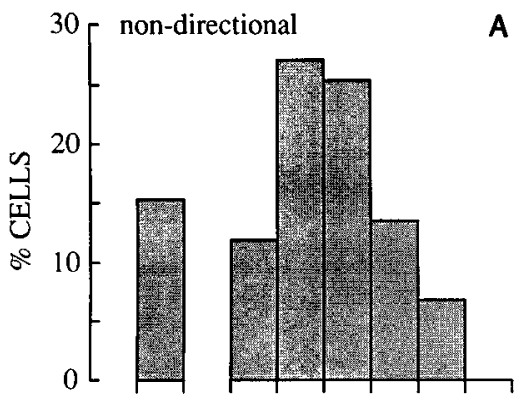

A
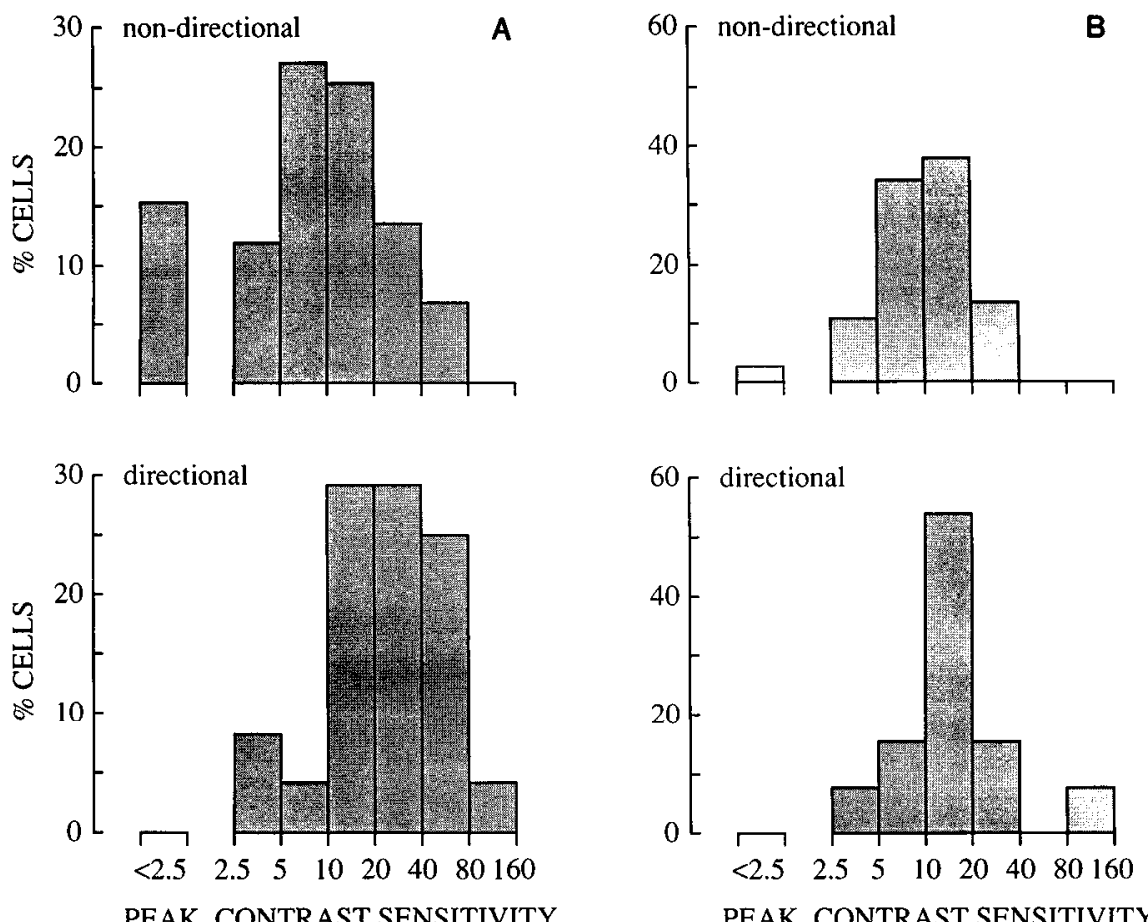

of those V1 cells that respond to achromatic stimuli show some directional preference.

\section{Contrast sensitivity and spatial frequency}

The contrast sensitivity measurements were fitted with a model of the receptive field based on difference of Gaussian functions (Hawken and Parker, 1987), and from this fitted function we obtained the peak contrast sensitivity and peak spatial frequency for each cell. Figure 5 shows the spatial contrast sensitivity functions obtained from the 2 neurons whose orientation and directional properties are shown in Figures 1 and 2 . Both cells are sensitive to a limited range of spatial frequencies and have clearly defined peaks; this bandpass feature is characteristic of most V1 neurons (DeValois et al., 1982b).

There is a considerable range of peak contrast sensitivities shown by our sample of neurons (Fig. 6), spanning about 1.5 $\log$ units for cells whose receptive fields are within $1.5^{\circ}$ of the fovea (Fig. 6A). All but one of the most sensitive cells in the foveal group have contrast thresholds between $1.5 \%$ and $2.5 \%$ (contrast sensitivities between 40 and 80 ), while the most insensitive cells require at least $40 \%$ contrast to evoke a response above threshold (sensitivities $<2.5$ ). The range of contrast sensitivities seems to be more restricted for neurons in the perifoveal group (Fig. 6B), where all cells, but one, have contrast thresholds $>2.5 \%$ and $<40 \%$. The relative reduction in sensitivity with increasing eccentricity parallels the psychophysical observation that there is a decrease in the contrast sensitivity of human observers, for a range of spatial frequencies, as the stimulus is positioned at increasing distances from the fixation point (Robson and Graham, 1981).

The foveal and perifoveal cells can be further divided into directional (directional preference $>2$ ) and nondirectional groups (see Figs. 6,7). The distribution of peak contrast sensitivities, especially for the foveal group (Fig. $6 \mathrm{~A}$ ), is shifted or skewed towards the higher sensitivity groups. The difference between the directional and nondirectional distributions for the foveal cells is significant (ANOVAR $F=19.1$; df 1,$81 ; p \ll$ 0.001 ). There is also a suggestion of a difference between the directional and nondirectional distributions for the perifoveal cells (ANOVAR $F=3.53$; df 1,$48 ; p<0.07$ ). For both foveal and perifoveal groups there are cells that show both directional selectivity and relatively high contrast sensitivity, which would be a prerequisite for supporting psychophysical performance.

The overall range of peak spatial frequencies for the cells covers at least 5 octaves (Fig. 7), and a considerable proportion of these cells have peak spatial frequencies in the range of 1.56.0 cycles/degree. These results are in agreement with those found by DeValois et al. (1982b) for V1 neurons with receptive fields at similar retinal eccentricities. Neurons with the highest peak spatial frequency are located in the foveal group (6-12 cycles/degree in Fig. $7 A$ ), while the more eccentric group does not have any cells with peak spatial frequencies above 6 cycles/ degree (Fig. $7 B$ ). The difference in peak spatial frequencies between the foveal and perifoveal groups is highly significant (ANOVAR $F=16.8 ; d f 1,119 ; p \ll 0.001)$. For 10 cells ( 8 in the foveal group and 2 in the perifoveal), we could determine orientation tuning and spatial frequency response tuning functions, but the contrast sensitivity tracking routine was unable to measure a contrast sensitivity function because the cell failed to respond reliably above the criterion to the highest contrast. Therefore, these cells are included in the group with sensitivities of $<2.5$, but peak spatial frequency could not be ascertained from the contrast sensitivity function, so they have not been included in the spatial frequency distribution.

There seems to be a small but consistent underrepresentation of the highest peak frequencies among the directionally tuned cells, in both the foveal and perifoveal cells. The directional and nondirectional distributions for both eccentricities show weakly significant differences (foveal: ANOVAR $F=4.3 ; d f 1,73 ; p<$ 0.05 ; perifoveal: ANOVAR $F=5.01 ; d f 1,46 ; p<0.05)$. We 

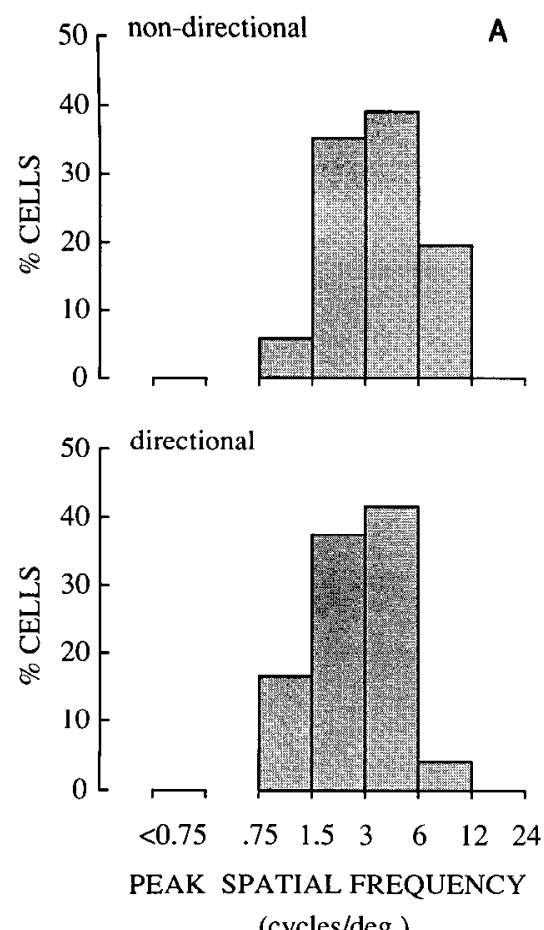

(cycles/deg.)
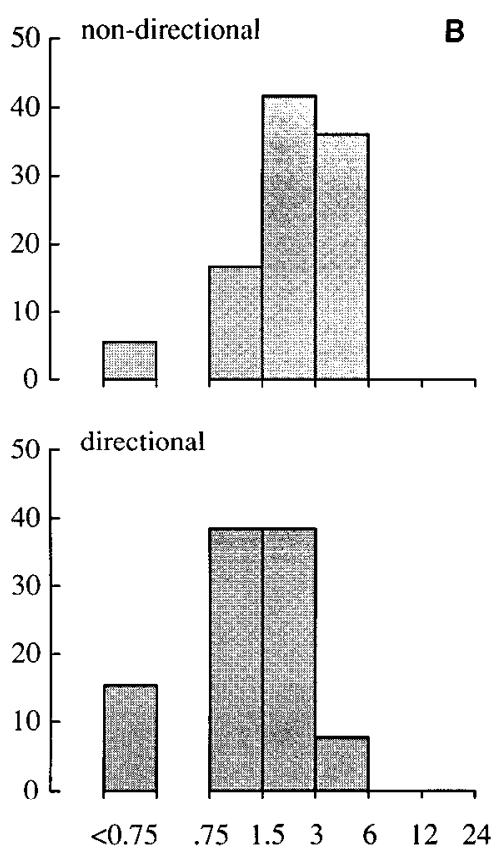

PEAK SPATIAL FREQUENCY

(cycles/deg.)
Figure 7. Distribution of optimal spatial frequency of directionally selectivc cells compared with the nondirectional cells. $A$, Cells with receptive field centers within $1.5^{\circ}$ of the fovea. $B$, Cells with fields $1.5^{\circ}-3.5^{\circ}$ eccentric. also assessed the grouped differences from the directional and nondirectional populations. As for the individual results above, the 2 populations are significantly different (ANOVAR $F=4.72$; df 2,$119 ; p<0.02$ ).

\section{Discussion}

In V1 there is selective segregation of the input terminals from the parvo- and magnocellular layers of the LGN and the maintenance of this segregation of inputs is reflected by the different functional properties exhibited by cells in the layers $4 \mathrm{c} \alpha$ and $4 c \beta$ (Blasdel and Fitzpatrick, 1984; Hawken and Parker, 1984). Therefore, as well as the prominent columnar arrangements in V1 (Hubel and Wiesel, 1977), there are substantial laminar differences in neuronal properties. This is particularly evident when considering the distribution of directionally selective cells in $\mathrm{V} 1$, which are found much more commonly in the upper portions of layer $4(4 a, 4 b$, and $4 c \alpha$ ) and layer 6 than in other cortical layers. This result is in sharp distinction to the distribution of directionally selective cells in cat striate cortex, where directionally selective cells are relatively frequent in all cortical layers (Gilbert, 1977). In layer 4b, the results show both directional and nondirectional cells, which, combined with the recent anatomical evidence of a patchy distribution of cells projecting from layer 4b to MT (Shipp and Zeki, 1985), makes it tempting to speculate that these patches of cells projecting to MT may be those which show direction selectivity. At present there is no experimental evidence that bears upon the organization of functionaly distinct cell types within layer $4 \mathrm{~b}$ itself. However, there are directionally selective cells in the 2 layers adjacent to layer $4 \mathrm{~b}$. lavers $4 \mathrm{a}$ and $4 \mathrm{c} \alpha$ (Fig. 4). Neurons in $4 \mathrm{~b}$ and $4 \mathrm{c} \alpha$ have many functional propertics in common: they respond well to achromatic stimuli, many are selective or biased for stimulus orientation, they have relatively low contrast thresholds, and some are directional. The spiny stellate cells of $4 \mathrm{c} \alpha$ send short axon branches to terminate in layer $4 b$; in addition, there is some extension of lower $4 \mathrm{~b}$ neuron dendrites into the magnocellular recipient zone in $4 \mathrm{c} \alpha$ (Lund and Boothe, 1975; Fitzpatrick et al., 1985). Thus, either directly or indirectly, cells in $4 \mathrm{c} \alpha$ and $4 \mathrm{~b}$ potentially share the same input and exhibit a number of functional properties that are consistent with the properties of magnocellular neurons of the lateral geniculate.

It is quite consistent with the current notions of the nature of the psychophysical channels proposed to account for directional mechanisms in human vision to find that some directionally selective neurons have relatively high contrast sensitivities. Many cells with relatively high sensitivities are among the directional population in layers $4 \mathrm{c} \alpha$ and $4 \mathrm{~b}$, which is again compatible with an input derived from the magnocellular pathway (Blasdel and Fitzpatrick, 1984; Hawken and Parker, 1984). In addition, the relative paucity of directionally tuned cells with high peak spatial frequencies also suggests a basis for the loss of correspondence between detection and identification seen in human subjects at high spatial frequencies (Watson et al., 1980).

A consistent picture of the processing of motion information is beginning to emerge from neurophysiological investigations in conjunction with psychophysical studies of motion perception and computational modeling of the visual system. Functionally two levels for the processing of motion information can be identified. The first involves the extraction of motion components by orientation and spatial frequency selective bandpass filters with direction selectivity. The second stage involves a combination of the responses of these bandpass filters to extract more global characteristics of the motion flow field (Adelson and Movshon, 1982). Tentatively, the first stage can be identified with direction-selective neurons in V1, while at least a proportion of the neurons in MT exhibit some of the properties required for successful implementation of the second stage (Movshon et al., 1985). However, a comparison of the spatiotemporal range of interactions in $\mathrm{V} 1$ and MT receptive fields with those of the short-range motion mechanism (Braddick, 
1974; Baker and Braddick, 1985) has led to the suggestion by Mikami et al. (1986b) that both V1 and MT receptive fields might be required to account for the properties of the shortrange mechanism.

Our results emphasize the uniqueness of the motion pathway and the efficiency of its organization in the primate. The uniqueness of the motion pathway is made clear by the fact that directionally selective cells are hardly seen at all outside those layers of $\mathrm{V} 1$ that are known anatomically to project, either directly or indirectly, to MT. The efficiency of the motion pathway is evident in the relatively high luminance contrast sensitivities associated with the cells within the pathway. Indeed, many of the direction-selective cells are sufficiently sensitive that the psychophysical observer could rely on the outputs of just a few cells for analyzing the components of motion in the portion of the visual image served by the receptive fields of those cells.

\section{References}

Adelson, E. II., and J. A. Movshon (1982) Phenomenal coherence of moving visual patterns. Nature 300: 523-525.

Albright, T. D. (1984) Direction and orientation selectivity of neurons in visual area MT of the macaque. J. Neurophysiol. 52: 1106-1130.

Baker, C. L., and O. J. Braddick (1985) Eccentricity dependent scaling of the limits for short-range apparent motion perception. Vision Res. 25: 803-812.

Blasdel, G. G., and D. Fitzpatrick (1984) Physiological organization of layer 4 in the macaque striate cortex. J. Neurosci. 4: 880-895.

Blasdel, G. G., and J. S. Lund (1983) Terminations of afferent axons in macaque striate cortex. J. Neurosci. 3: 1389-1413.

Braddick, O. J. (1974) A short-range process in apparent movement. Vision Res. 14: 519-527.

DeValois, R. L., E. W. Yund, and N. Hepler (1982a) The orientation and direction selectivity of cells in the macaque visual cortex. Vision Res. 22: 531-544.

DeValois, R. L., D. G. Albrecht, and L. G. Thorell (1982b) Spatial frequency selectivity of cells in the macaque visual cortex. Vision Res. 22: $545-559$.

Dow, B. M. (1974) Functional classes of cells and their laminar distribution in monkey visual cortex. J. Neurophysiol. 37: 927-946.

Dubner, R., and S. M. Zeki (1971) Response properties and receptive fields of cells in an anatomically defined region of the superior temporal sulcus in the monkey. Brain Res. 35: 528-532.

Fitzpatrick, D., J. S. Lund, and G. G. Blasdel (1985) Intrinsic connections of the macaque striate cortex: Afferent and efferent connections of lamina 4C. J. Neurosci. 5: 3329-3349.

Fries, W., K. Keizer, and H. G. J. M. Kuypers (1985) Layer VI cells in the macaque striate cortex project to both supcrior colliculus and prestriate visual area V5. Exp. Brain Res. 58: 613-616.

Gilbert, C. D. (1977) Laminar differences in receptive field properties of cells in cat primary visual cortex. J. Physiol. (Lond.) 268: $391-$ 421.

Hawken, M. J., and A. J. Parker (1984) Contrast sensitivity and orientation selectivity in laminar IV of the striate cortex of Old World monkeys. Exp. Brain Res. 54: 367-372.

Hawken, M. J., and A. J. Parker (1987) Spatial properties of neurons in the monkey striate cortex. Proc. R. Soc. Lond. [Biol.] 231: 251288.

Hendrickson, A. E., J. R. Wilson, and M. P. Ogren (1978) The neuroanatomical organization of pathways between the dorsal lateral geniculate nucleus and the visual cortex in old and new world primates. J. Comp. Neurol. 182: 123-136.

Hubel, D. H., and T. N. Wiesel (1968) Receptive fields and functional architecture of monkey striate cortex. J. Physiol. (Lond.) 195: 215243.

Hubel, D. H., and T. N. Wiesel (1972) Laminar and columnar distribution of geniculo-cortical fibres in macaque monkey. J. Comp. Neurol. 146: 421-450.

Hubel, D. H., and T. N. Wiesel (1977) Functional architecture of macaque monkey visual cortex. Proc. R. Soc. Lond. [Biol.] 198: 159.
Levinson, E., and R. W. Sekuler (1975) The independence of channels in human vision selective for the direction of movement. J. Physiol. (Lond.) 250: 347-366.

Livingstone, M. S., and D. H. Hubel (1984) Anatomy and physiology of a color system in the primate visual cortex. J. Neurosci. 4: 309356.

Lund, J. S., and R. G. Boothe, (1975) Interlaminar connections and pyramidal neuron organization in the visual cortex, area 17 , of the macaque monkey. J. Comp. Neurol. 159: 305-334.

Lund, J. S., R. D. Lund, A. E. Hendrickson, A. H. Bunt, and A. F. Fuchs (1976) The origin of efferent pathways from the primary visual cortex, area 17, of the macaque monkey as shown by retrograde transport of horseradish peroxidase. J. Comp. Neurol. 164: 287-304.

Maunsell, J. H. R., and D. C. Van Essen (1983) The connections of the middle temporal visual area (MT) and their relationship to cortical hierarchy in the macaque monkey. J. Neurosci. 3: 2563-2586.

Merrill, E. G., and A. Ainsworth (1972) Glass-coated platinum-plated tungsten microelectrodes. Med. Biol. Engin. 10: 662-672.

Mikami, A., W. T. Newsome, and R. H. Wurtz (1986a) Motion selectivity in macaque visual cortex. I. Mechanisms of direction and speed selectivity in extrastriate area MT. J. Neurophysiol. 55: 13081327.

Mikami, $\Lambda .$, W. T. Newsome, and R. H. Wurtz (1986b) Motion selectivity in macaque visual cortex. II. Spatiotemporal range of directional interactions in MT and V1. J. Neurophysiol. 55: 1328-1339.

Movshon, J. A., I. D. Thumpson, and D. J. Tolhurst (1978) Spatial summation in the receptive fields of simple cells in the cat's striate cortex. J. Physiol. (Lond.) 283: 53-77.

Movshon, J. A., E. H. Adelson, M. S. Gizzi, and W. T. Newsome (1985) The analysis of moving visual patterns. Exp. Brain Res. (Suppl.) 11: $117-151$.

Newsome, W. T., R. H. Wurtz, M. Dursteler, and A. Mikami (1985) Deficits in visual motion processing following ibotenic acid lesions of the middle temporal visual area of the macaque monkey. J. Neurosci. 5: $825-840$.

Orban, G. A., H. Kennedy, and J. Bullier (1986) Velocity sensitivity and dircction sclcctivity of neurons in areas $\mathrm{V} 1$ and $\mathrm{V} 2$ of the monkey: Influence of eccentricity. J. Neurophysiol. 56: $462-480$.

Pantle, A., and R. W. Sekuler (1968) Size-detecting mechanisms in human vision. Science 162: 1146-1148.

Parker, A. J., and M. J. Hawken (1988) Two-dimensional spatial structure of receptive fields in monkey striate cortex. J. Opt. Soc. Am. 5: 598-605.

Robson, J. G., and N. Graham (1981) Probability summation and regional variation in contrast sensitivity across the visual field. Vision Res. 21: 409-418.

Schiller, P. H., B. H. Finlay, and S. F. Volman (1976) Quantitative studies of single-cell properties in monkey striate cortex. I. Spatiotemporal organization of receptive fields. J. Neurophysiol. 39: 12881319.

Sckulcr, R. W., and L. Ganz (1963) A new aftereffect of seen motion with a stabilized retinal image. Science 139: 419-420.

Sharpe, C., and D. J. Tolhurst (1973) The effects of temporal modulation on the orientation channels of the human visual system. Perception 2: 23-29.

Shipp, S., and S. M. Zeki (1985) Segregated output to area V5 from layer $4 \mathrm{~b}$ of macaque monkey striate cortex. J. Physiol. (Lond.) 369: $32 \mathrm{P}$.

Van Essen, D. C., J. H. R. Maunsell, and J. L. Bixby (1981) The middle temporal visual area in the macaque: Myeloarchitecture, connections, functional properties and topographic organization. J. Comp. Neurol. 199: 293-326.

Watson, A. B., P. G. Thompson, B. J. Murphy, and J. Nachmias (1980) Summation and discrimination of gratings moving in opposite directions. Vision Res. 20: 341-347.

Wong-Riley, M. (1979) Changes in the visual system of monocularly sutured or enucleated cats demonstrable with cytochrome oxidase histochemistry. Brain Res. 171: 11-28.

Zeki, S. M. (1969) Representation of central visual fields in prestriate cortex of monkey. Brain Res. 14: 271-291.

Zeki, S. M. (1974) Functional organization of a visual area in the posterior bank of the superior temporal sulcus of the rhesus monkey. J. Physiol. (Lond.) 236: 549-573. 